Document downloaded from:

http://hdl.handle.net/10251/148013

This paper must be cited as:

Peng, G.; Albero-Sancho, J.; García Gómez, H.; Shalom, M. (2018). A Water-Splitting Carbon Nitride Photoelectrochemical Cell with Efficient Charge Separation and Remarkably Low Onset Potential. Angewandte Chemie International Edition. 57(48):15807-15811. https://doi.org/10.1002/anie.201810225

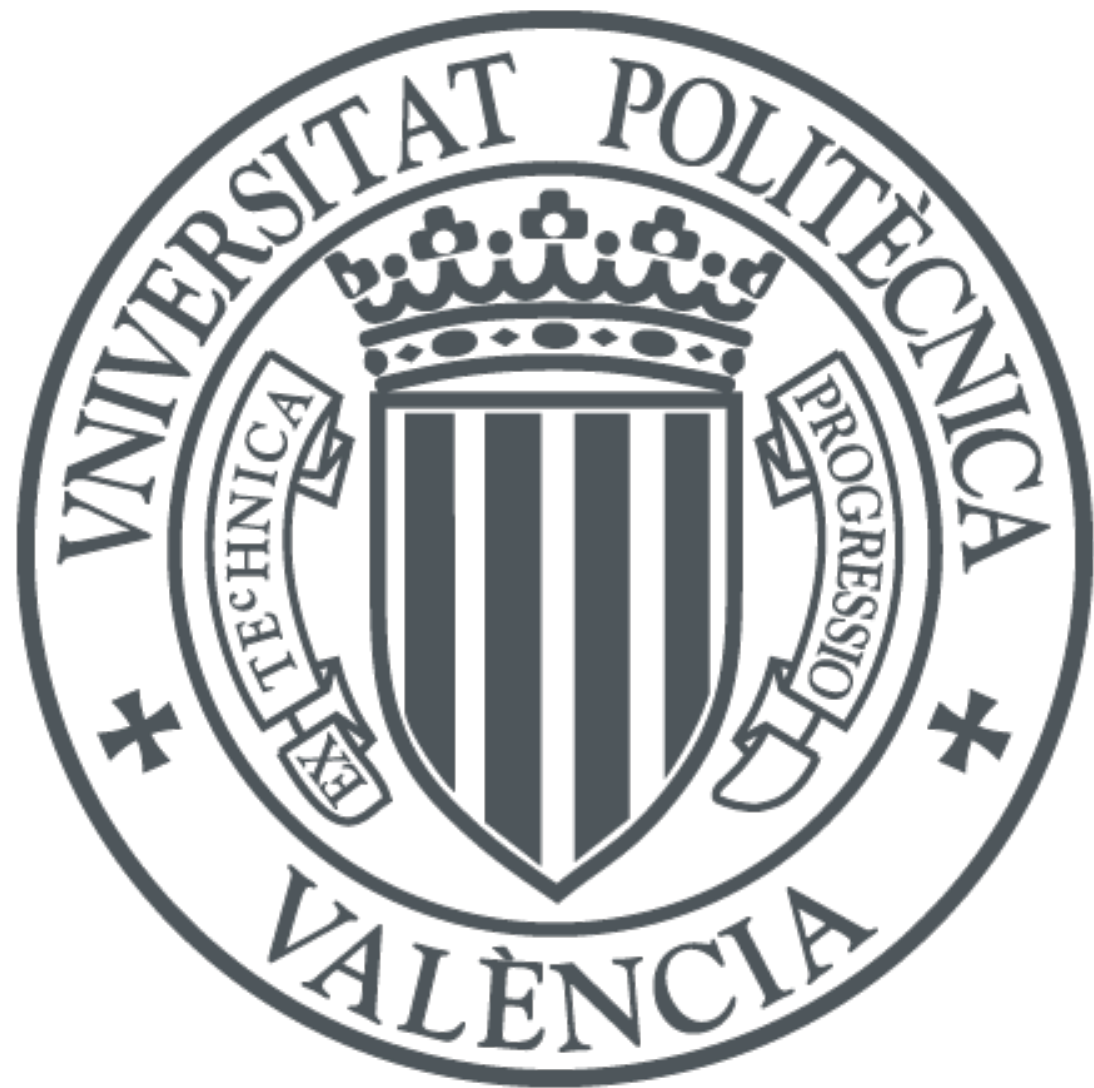

The final publication is available at

https://doi.org/10.1002/anie.201810225

Copyright John Wiley \& Sons

Additional Information

"This is the peer reviewed version of the following article: Peng, Guiming, Josep Albero, Hermenegildo Garcia, and Menny Shalom. 2018. A Water-Splitting Carbon Nitride Photoelectrochemical Cell with Efficient Charge Separation and Remarkably Low Onset Potential. Angewandte Chemie International Edition 57 (48). Wiley: 1580711. doi:10.1002/anie.201810225, which has been published in final form at https://doi.org/10.1002/anie.201810225. This article may be used for non-commercial purposes in accordance with Wiley Terms and Conditions for Self-Archiving." 


\title{
Water Splitting Carbon Nitride Photoelectrochemical Cell with Efficient Charge Separation and Remarkably Low Onset Potential
}

Guiming Peng, ${ }^{1,2}$ Josep Albero, ${ }^{3}$ Hermenegildo Garcia ${ }^{3}$, Menny Shalom ${ }^{1 *}$

${ }^{1}$ Department of Chemistry and Ilse Katz Institute for Nanoscale Science and Technology, Ben-Gurion University of the Negev Beer-Sheva 8410501, Israel.

${ }^{2}$ School of Materials and Energy, Guangdong University of Technology, Guangzhou 510006, China.

${ }^{3}$ Instituto mixto de tecnología química (CSIC-UPV), Universitat Politècnica de València, Avda de los Naranjos s/n E-46022, Valencia, Spain.

\begin{abstract}
Carbon nitride $(\mathrm{CN})$ is considered a promising semiconductor for water-splitting photoelectrochemical cells owing to its low price, tunable band gap, suitable energyband position, stability to harsh chemical conditions and environmentally benignity. However, despite $\mathrm{CN}$ promising performance in various fields such as photo-, and electrocatalysis, its exploitation in photoelectrochemical cells is hindered by the limited poor charge-separation and hole-extraction efficiency of the unordered structures which are the result of current deposition methods. Here we report a simple method to grow a closely-packed, crystalline $\mathrm{CN}$ layer directly on a conductive substrate. A layer of melamine is first grown on a pre-seeded conductive substrate by the nucleated growth of melamine crystals. Upon calcination, the melamine crystals turn into a highly-ordered, low-defect $\mathrm{CN}$ layer, which is intimately connected to the conductive substrate. The $\mathrm{CN}$ film exhibits excellent charge-separation properties. detailed (photo)electrochemical and transient absorption measurements indicate an efficient hole extraction (up to 50\%), a long electron lifetime and a lack of defect states below the $\mathrm{CN}$ conduction band. Consequently, the $\mathrm{CN}$ photoanode exhibits a
\end{abstract}


markedly low overpotential of $0.25 \mathrm{~V}$ versus reversible hydrogen electrode (RHE), which is comparable with the state-of-the-art metal-based photoanodes, a good photocurrent density of $116 \mu \mathrm{A} / \mathrm{cm}^{2}$ at $1.23 \mathrm{~V}$ versus RHE in an alkaline solution without sacrificial agent, as well as excellent stability over a wide $\mathrm{pH}$ range (0-14). This work opens new opportunities in photoelectrochemistry and other energy-related applications by illustrating a method for the facile growth of ordered metal-free $\mathrm{CN}$ and other 2D materials on conductive substrates.

\section{Introduction}

Graphitic carbon nitride $(\mathrm{CN})$ exhibits promising photo- and electrocatalytic activity for various reactions, such as water splitting, ${ }^{1-8}$ oxygen reduction, ${ }^{9-12}$ and $\mathrm{CO}_{2}$ reduction, ${ }^{13-18}$ derived from its low price, tunable electronic and catalytic properties, environmentally friendliness, and excellent stability under harsh conditions. However, its exploitation in (photo)electronic devices has been limited by (i) the difficulty in coating a conductive substrate with a high-quality, homogenous and closely-packed crystalline $\mathrm{CN}$ layer, and (ii) the poor charge separation and fast recombination in $\mathrm{CN}$ materials, owing to poor electron and hole mobility, sluggish hole extraction, and large amounts of structural defects. ${ }^{19}$ In the last years, great progress in the growth of $\mathrm{CN}$ layers on various substrates has been made using various methods, such as thermal vapor condensation, ${ }^{20-22}$ liquid microcontact printing, ${ }^{23}$ doctor-blade technique, ${ }^{24-27}$ sol processing, ${ }^{28}$ and more. ${ }^{29,}{ }^{30}$ Moreover, $\mathrm{CN}$ has been successfully used as adsorber in photoelectrochemical cells (PECs), ${ }^{20-23,} 25,31$ organic light-emitting diodes, ${ }^{32-34}$ and solar cells. ${ }^{29},{ }^{35-37}$ However, despite the progress in utilizing $\mathrm{CN}$ in PECs, ${ }^{31}$ several 
important factors still limit its efficiency. One major limitation lies on the poor charge conductivity through the layer. ${ }^{19,38}$ The low conductivity results in increased charge recombination and low PEC performance. The insufficient charge separation may stem from the fact that the current growth methods usually lead to an unordered layered structure with big grain boundaries. In 2015, it was demonstrated that, in contrast to graphene, electron and hole mobility within $\mathrm{CN}$ materials is perpendicular to the $2 \mathrm{D}$ layer. ${ }^{38}$ This study showed that if the $\mathrm{CN}$ layers are close and ordered enough, the electron and hole mobility is significantly enhanced. Moreover, in a recent study, Durrant et al. reported that $\mathrm{CN}$ powder contains deep defect levels up to $0.3 \mathrm{eV}$ below its conduction bands, which act as traps for electrons, thus deteriorating the total photocatalytic activity. ${ }^{39}$ Therefore, we envision that the synthesis of continuous crystalline $\mathrm{CN}$ films with fewer defects will result in a significant improvement in their (photo)electrocatalytic performance.

Herein we demonstrate a simple and versatile method to grow crystalline $\mathrm{CN}$ films with a closely-packed layered structure on fluorine-doped tin oxide glass (FTO), via the seeded crystallization of $\mathrm{CN}$ monomers followed by their calcination at high temperature. The growth of $\mathrm{CN}$ monomer crystals was enabled by the deposition of nucleation sites on FTO prior to the growth of crystals from supersaturated monomer solutions. Upon calcination, a strongly bonded, defect-free crystalline CN layer on FTO was successfully obtained. The CN film exhibited impressive PEC performance with a photoanodic photocurrent of $116 \mu \mathrm{A} / \mathrm{cm}^{2}$ at $1.23 \mathrm{~V}$ vs RHE and up to a $1-\mathrm{V}$ shift of the onset potential under one 1 sun in $0.1 \mathrm{M} \mathrm{KOH}$ aqueous solution. Detailed 
electrochemical and time-resolved absorption spectroscopy investigations prove that the excellent PEC performance is a result of improved electron transport, fewer defects, and favorable hole-extraction properties.

\section{Results and discussion}

A schematic illustration of the growth of a melamine film on FTO is shown in Figure 1a. Melamine crystals were deposited on FTO glass via a two-step process. First, in order to initiate the crystallization of melamine on FTO, we deposited melamine seeds on its surface by dipping a FTO glass slide into a saturated melamine solution (in methanol) (Figure S1 and S2). Scanning electron microscope (SEM) image of the surface of the slide after dipping in a saturated methanolic melamine solution shows that the melamine seeds were homogeneously deposited on FTO (Figure S2). Next, the seeded FTO was soaked into a hot aqueous solution that was supersaturated with melamine to prevent the dissolution of the melamine seeds. Upon cooling, as a result of the decrease in solubility (from $\sim 3.4 \mathrm{~g}$ at $95{ }^{\circ} \mathrm{C}$ to $\sim 0.4 \mathrm{~g}$ at $25^{\circ} \mathrm{C}$, Figure $\mathrm{S} 3$ ), the pre-deposited melamine seeds on FTO serve as nucleation sites for the crystallization of melamine from solution. Figure S4 shows that melamine molecules started to crystalize on the modified FTO and the FTO color changed immediately after immersion into the hot, supersaturated melamine solution $\left(95^{\circ} \mathrm{C}\right)$. After cooling to 25 ${ }^{\circ} \mathrm{C}$, a uniform layer of melamine crystals on FTO was obtained (Figure 1b-d and S4a). We note that melamine molecules cannot be crystalized on bare FTO without the seeding process (Figure S4b), emphasizing the importance of the seeding step. Furthermore, the melamine film can be grown from supersaturated solutions at different starting temperatures due to the higher solubility compared to that at room temperature (Figure S5). SEM images of the melamine layer on FTO reveal that the $\mathrm{CN}$ layer 
adopted a polyhedral morphology (Figure 1c, d), which is different to that of the pristine powder (Figure S6). The melamine crystals are intimately connected to the FTO with a layer thickness of $\sim 40 \mu \mathrm{m}$ (Figure 1c, d, and S7). XRD measurements of the melamine film and pristine powder show similar crystal structures. However, the relative intensity of some peaks is different (Figure 1e), owing to the directionality of melamine crystals which is directed by the nucleation sites on the FTO glass.
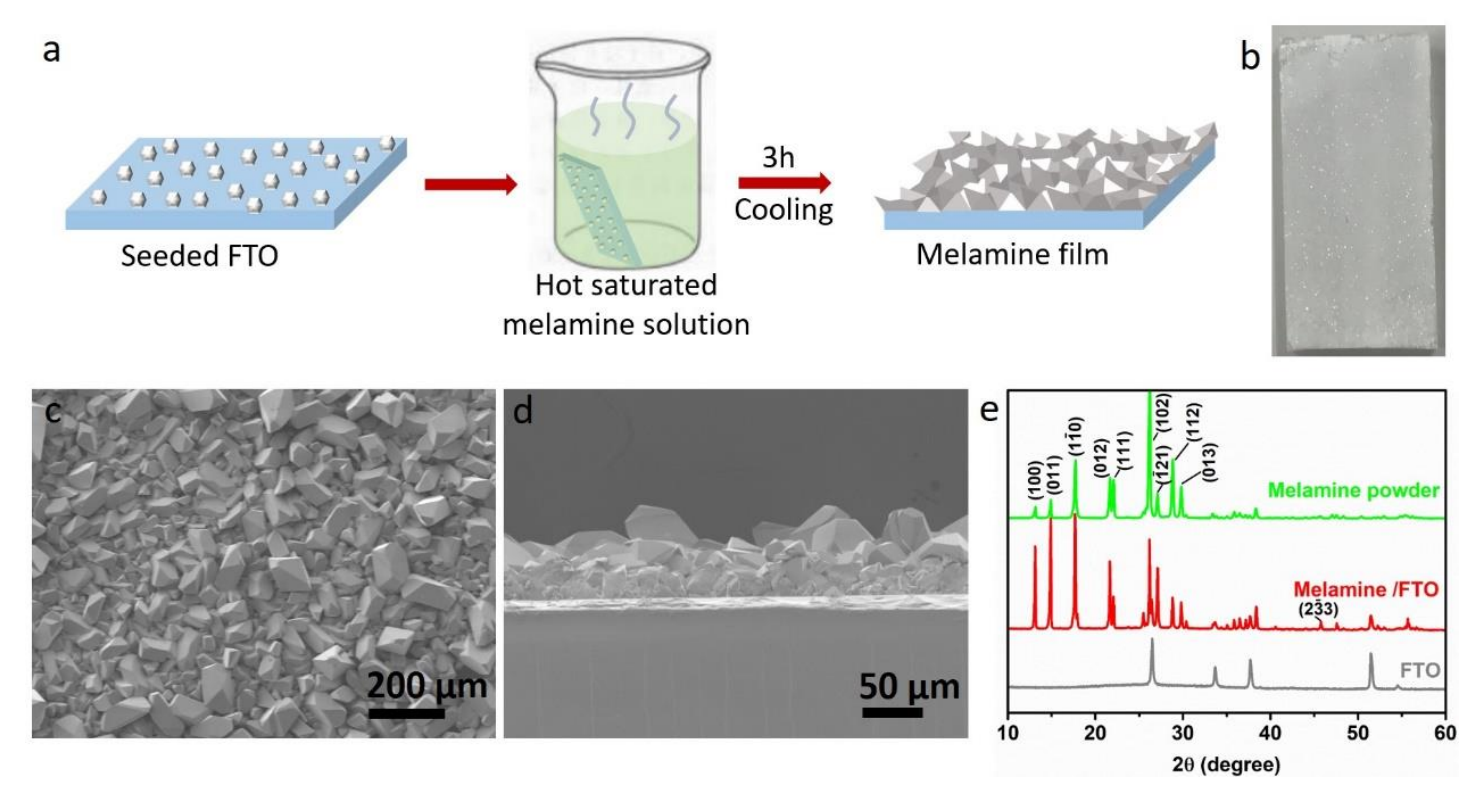

Figure 1. (a) Schematic illustration of the melamine film fabrication. (b) A digital photo of the melamine film on FTO. (c) Top-view SEM image of the melamine film. (d) Cross-sectional SEM image of the melamine film. (e) XRD patterns of melamine film on FTO and melamine powder.

The $\mathrm{CN}$ film was obtained by calcining the melamine film at $550{ }^{\circ} \mathrm{C}$ for 4 hours, leading to a uniform yellow CN layer on FTO glass (Figure 2a). The strong fluorescence under illumination $(370 \mathrm{~nm})$ suggests that the layer is smooth, without many defects. The $\mathrm{CN}$ film is composed of a relatively dense internal layer $(\sim 20 \mu \mathrm{m})$ below an external upper layer of flower-like CN microstructures (Figure S8). The dense layer, closer to the FTO, is composed of interconnected porous $\mathrm{CN}$ networks (Figure $2 \mathrm{~b}$ and $\mathrm{S} 8 \mathrm{~b})$. It is noted that the morphology of the $\mathrm{CN}$ film is different from $\mathrm{CN}$ powder 
obtained by calcination of melamine powder (Figure S9). Strong sonication of the CN film for 5 minutes results in the selective removal of the upper microstructures, while the compact layer in contact with the FTO remains intact. This demonstrates that the dense $\mathrm{CN}$ layer is strongly attached to the FTO, while the uppermost microstructures are not (Figure $2 \mathrm{~b}$ and $2 \mathrm{c}$ ). As a control experiment, a $\mathrm{CN}$ film obtained by the calcination of melamine powder, spread on pristine FTO was easily peeled off (Figure S10), indicating the importance of the seeding step before the film growth. In addition, the extension of the proposed method to other substrates and monomers is exemplified by the growth of $\mathrm{CN}$ on silica glass (Figure S11), by the coating of FTO with a carbonrich $\mathrm{CN}$ layer using barbituric acid as the precursor (Figure S12), and by the crystallization of cyanuric acid on FTO (Figure S13).
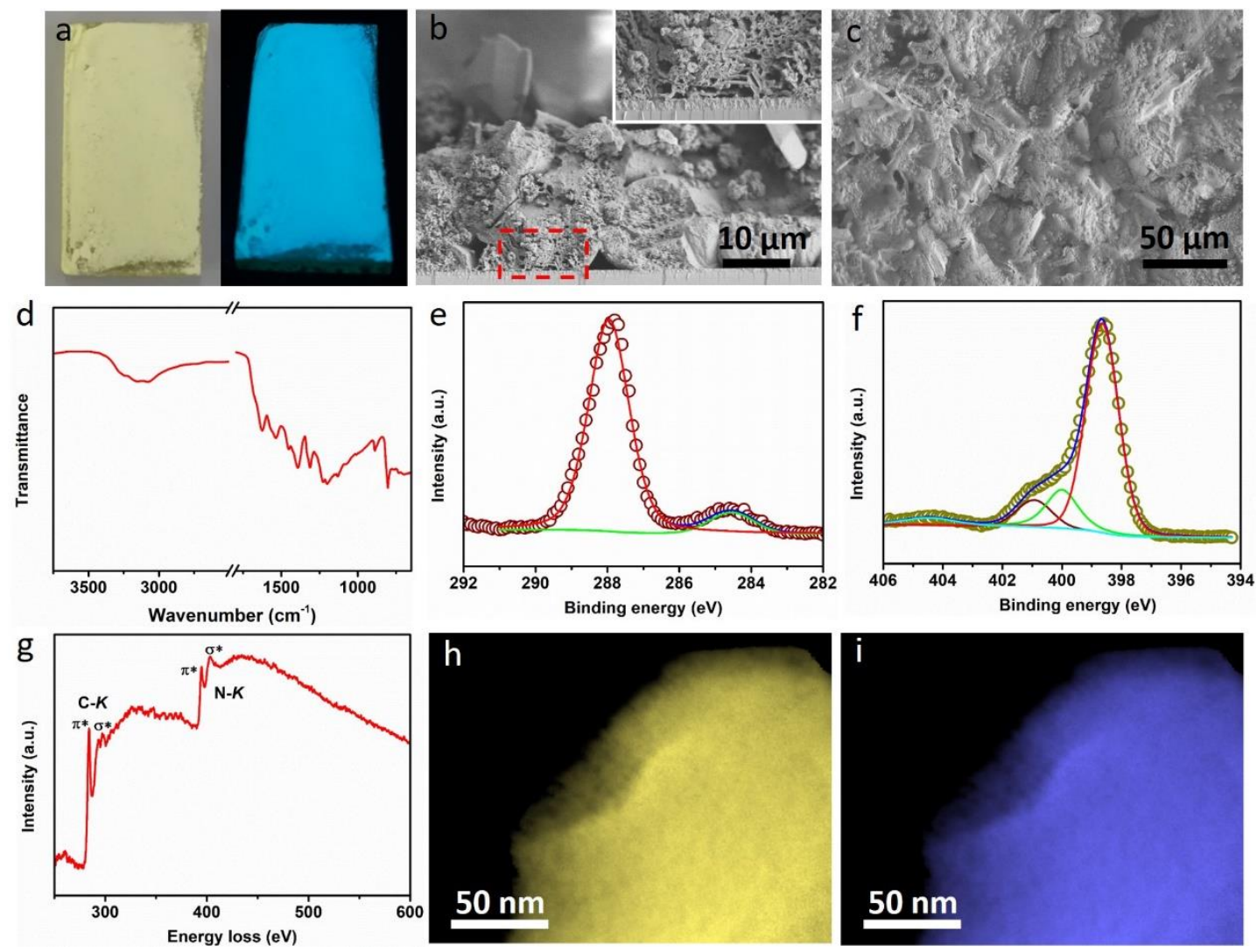
Figure 2. Characterizations of the $\mathrm{CN}$ film on FTO. (a) Optical images of $\mathrm{CN}$ film with and without illumination at $365 \mathrm{~nm}$. (b) Cross-section and (c) top-view SEM image of the $\mathrm{CN}$ film after 5 minutes sonication. The inset is a magnified SEM image of the squared area. (d) FTIR spectra. (e) N1s XPS and (f) C1s XPS spectra. (g) EELS spectra of CN film. (h) EDS element mapping of carbon and (i) nitrogen.

Fourier transform infrared spectra (FTIR) of the $\mathrm{CN}$ film show the typical stretching modes of CN heterocycles in the range of $1200-1700 \mathrm{~cm}^{-1}$ (Figure $2 \mathrm{~d}$ ). ${ }^{40-42}$ The peak at $802 \mathrm{~cm}^{-1}$ is attributed to the breathing mode of the triazine unit. ${ }^{24,40}$ The chemical states of the CN film were studied by X-ray photoelectron spectroscopy (XPS). In the C1s spectrum, two peaks at 284.6 and $288 \mathrm{eV}$ were assigned to $\mathrm{sp}^{2} \mathrm{C}$ in carbonaceous environment and $\mathrm{sp}^{2} \mathrm{C}$ in $\mathrm{C}-\mathrm{N}$ heterocycles, respectively (Figure $2 \mathrm{e}$ ). ${ }^{25,}, 43$ The $\mathrm{C} 1 \mathrm{~s}$ spectrum of the $\mathrm{CN}$ powder obtained by calcination of melamine powder (Figure S14) reveals a signal for $\mathrm{sp}^{2} \mathrm{C}-\mathrm{N}$ bonds that is similar to that observed for $\mathrm{CN}$ on $\mathrm{FTO}$, but with a significantly stronger $\mathrm{sp}^{2} \mathrm{C}=\mathrm{C}$ peak. The $\mathrm{C}=\mathrm{C}$ bonds are attributed to defect sites in the $\mathrm{CN}$ structure, which are formed during the degradation of melamine upon heating. Lower percentage of $\mathrm{C}$ atoms forming $\mathrm{C}=\mathrm{C}$ bonds in the $\mathrm{CN}$ films implies stronger intermolecular interactions within the melamine crystals which impede the degradation of melamine, leading to higher quality $\mathrm{CN}$ layers with negligible defects. The N1s spectra support the $\mathrm{CN}$ formation and best deconvolution of the N1s peak indicates the existence of three peaks at $398.7,400,400.9 \mathrm{eV}$, which correspond well to $\mathrm{C}=\mathrm{N}-\mathrm{C}, \mathrm{N}$ $\mathrm{C}_{3}$, and $\mathrm{C}-\mathrm{NH}-\mathrm{C}$ and $\mathrm{C}-\mathrm{NH}_{2}$ (Figure 2f), respectively. ${ }^{43}$ Electron energy loss spectroscopy (EELS) proves the formation of a $\mathrm{sp}^{2} \mathrm{C}=\mathrm{N}$ network by revealing the presence of $1 \mathrm{~s}-\pi^{*}$ and $1 \mathrm{~s}-\sigma^{*}$ transitions in both the carbon $\mathrm{K}$ edge and the nitrogen $\mathrm{K}$ edge (Figure 2g). ${ }^{44}$ Energy dispersive X-ray spectroscopy (EDS) element mappings shows a uniform distribution of $\mathrm{C}$ and $\mathrm{N}$ within the $\mathrm{CN}$ layer (Figure $2 \mathrm{~h}$ and $2 \mathrm{i}$ ). 

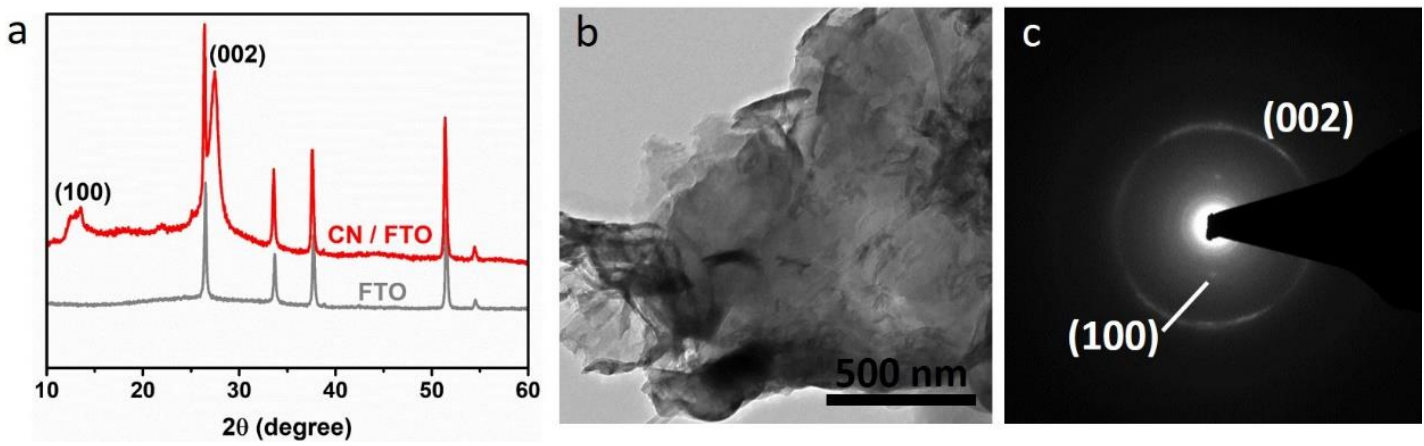

Figure 3. (a) XRD diffraction pattern of CN layer on FTO (b) TEM image, and (c) SAED pattern of $\mathrm{CN}$ layer.

The crystal structure of the $\mathrm{CN}$ layer was characterized by XRD and selected area electron diffraction (SAED). The XRD pattern of the $\mathrm{CN}$ film shows one sharp, strong interplanar stacking peak (002) at $27.5^{\circ}$ and a relative weak in-plane (100) peak at $13.4^{\circ}$ (Figure 3a). ${ }^{40}$ The strong XRD peak at $27.5^{\circ}$ indicates a good layer organization. The layered structure with relatively smooth surface compared to the $\mathrm{CN}$ powder is observed by transmission electron microscopy (TEM) image (Figure 3b, S9). SAED patterns (Figure 3c and S15) show the (002), (100) diffractions, further supporting a crystalline state for the $\mathrm{CN}$ layer. ${ }^{45}$ However, the diffraction fringes of the $\mathrm{CN}$ film could not be obtained due to the instability of such $\mathrm{CN}$ upon exposure to the electron beam (Figure S16).

The UV-vis spectrum of the CN film indicates that the absorption onset is at $\sim 475$ $\mathrm{nm}$ (corresponding to a bandgap of $2.61 \mathrm{eV}$ ), i.e. slightly red-shifted compared to the one of the $\mathrm{CN}$ powder ( 460 nm) (Figure S17). The red shift is likely due to the dense $\mathrm{CN}$ layer compared to the $\mathrm{CN}$ powder (Figure S18). In consistence with this, the fluorescence spectrum of the $\mathrm{CN}$ film is red-shifted relative to that of the $\mathrm{CN}$ powder film (Figure S19). The sharp absorption edge implies that the layer contains a low number of structural defects. Mott-Schottky measurements combined with the UV-vis spectrum of the $\mathrm{CN}$ layer reveal that the conduction band (flat band) and the valence 
band are located at -0.75 and $1.86 \mathrm{eV}$ vs RHE, respectively, which is suitable for water reduction and oxidation (Figure S20).

The photoelectrochemical properties of the $\mathrm{CN}$ film were measured in a typical three-electrodes cell, using $0.1 \mathrm{M} \mathrm{KOH}$ as the electrolyte, $\mathrm{Pt}$ as the counter electrode and $\mathrm{Ag} / \mathrm{AgCl}$ as the reference electrode. Linear sweep voltammetry (LSV) curves of the $\mathrm{CN}$ electrode in the dark and under one-sun illumination demonstrate a typical PEC behavior with an onset potential at only $0.25 \mathrm{~V}$ vs RHE (Figure 4a). To the best of our knowledge, this potential is the lowest among $\mathrm{CN}$ electrodes and comparable to stateof-the-art materials, such as $\mathrm{BiVO}_{4}{ }^{46-48}$ The low onset potential indicates that, upon illumination, the production of photocurrent starts almost 1 volt below the water oxidation potential. Naturally, the photocurrent increased at higher potentials, reaching $116 \mu \mathrm{A} / \mathrm{cm}^{2}$ at $1.23 \mathrm{~V}$ vs. RHE (Figure $4 \mathrm{~b}$ ). This value is among the best reported for CN electrodes without sacrificial agent (Table S2) and it is $>200$ times higher than what is observed with a CN powder electrode $\left(\sim 0.5 \mu \mathrm{A} / \mathrm{cm}^{2}\right.$, Figure S18 and S21). Durability test revealed that the $\mathrm{CN}$ can maintain the photocurrent for $30 \mathrm{~min}$, and with $\sim 70 \%$ photocurrent retained after one hour (Figure S22). In addition, the changes in the photocurrent of the $\mathrm{CN}$ electrode after the removal of the flower-like top microstructures is negligible (Figure S23), indicating that the underlayer continuous $\mathrm{CN}$ film is the main contributor to the photocurrent. Incident photon-to-current conversion efficiency (IPCE) measurements of the $\mathrm{CN}$ electrode at different wavelengths show a direct correlation between light absorption and photocurrent. The photocurrent onset is at $\sim 70 \mathrm{~nm}$ and the IPCE values at $400 \mathrm{~nm}$ and $420 \mathrm{~nm}$ reach $8.5 \%$ and $3.6 \%$, respectively (Figure $4 \mathrm{c}$ ).

An important advantage of $\mathrm{CN}$ over other semiconductors is its high chemical stability in solution over a wide range of pHs. LSV curves upon on/off light illumination 
of the $\mathrm{CN}$ electrode in $0.1 \mathrm{M} \mathrm{NaH}_{2} \mathrm{PO}_{4}$ buffer $(\mathrm{pH} 7)$ and $0.5 \mathrm{M} \mathrm{H}_{2} \mathrm{SO}_{4}(\mathrm{pH} 0.2)$ aqueous solution show high, stable photocurrents (Figure 4d). The photocurrent at $1.23 \mathrm{~V}$ vs RHE in neutral electrolyte reaches $71 \mu \mathrm{A} / \mathrm{cm}^{2}$, while that in acid is $64 \mu \mathrm{A} / \mathrm{cm}^{2}$ despite a water oxidation rate that is lower than in alkaline solution. Notably, the onset potential in both electrolytes is below $0.3 \mathrm{~V}$ vs RHE. The good photocurrents together with the low onset potentials in various electrolytes denote its promising potential in PEC water splitting.
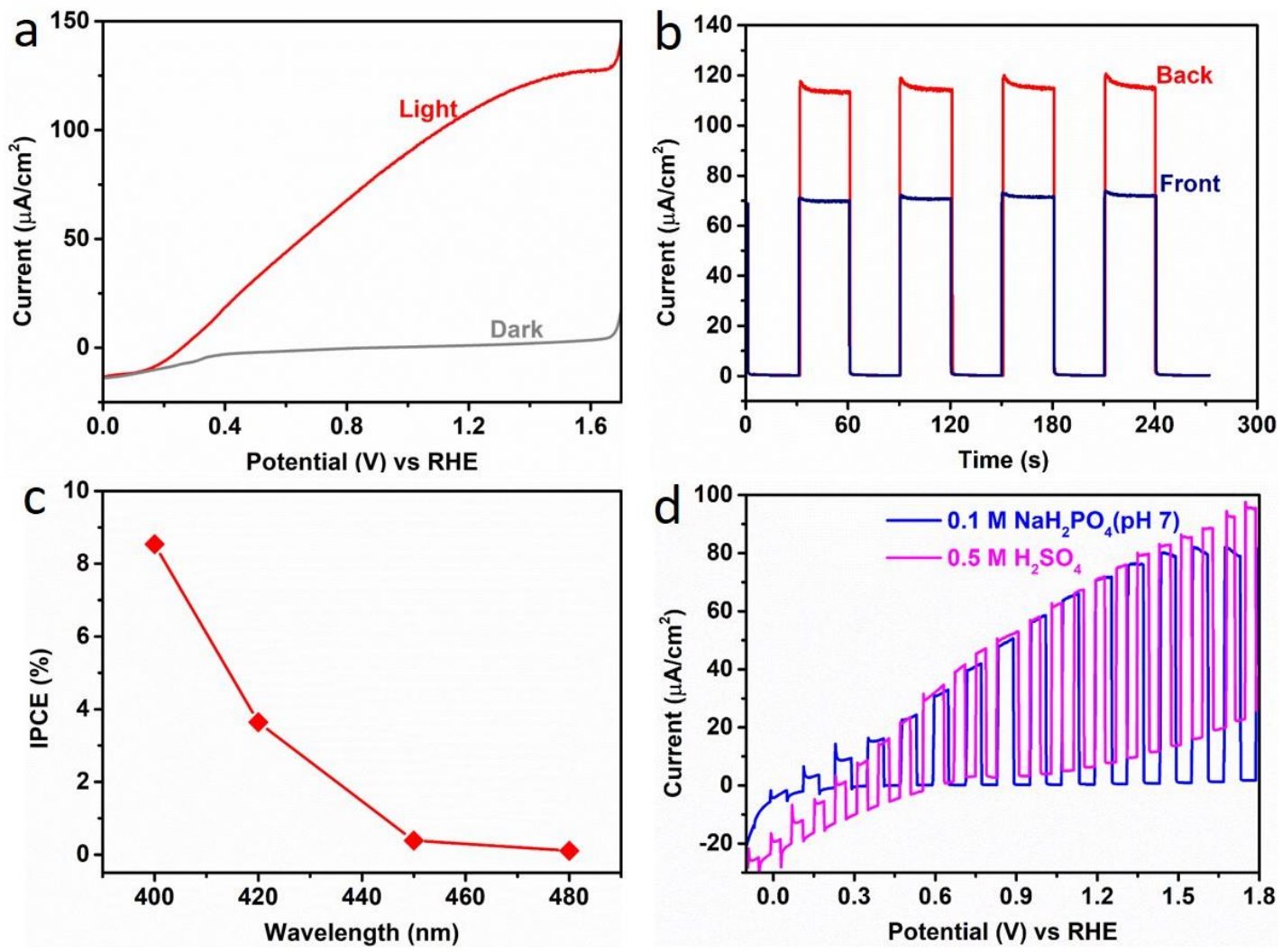

Figure 4. (a) LSV curves of the $\mathrm{CN}$ film in $0.1 \mathrm{M} \mathrm{KOH}$ aqueous solution with and without one sun illumination. (b) Photocurrent of the $\mathrm{CN}$ film in $0.1 \mathrm{M} \mathrm{KOH}$ aqueous solution at $1.23 \mathrm{~V}$ vs RHE upon illumination of one sun. (c) IPCE plot of the CN film electrode as a function of wavelength. (d) LSV curves of the $\mathrm{CN}$ film in $0.1 \mathrm{M} \mathrm{NaH}_{2} \mathrm{PO}_{4}$ ( $\mathrm{pH} 7$ ) and $0.5 \mathrm{M} \mathrm{H}_{2} \mathrm{SO}_{4}$ aqueous solution upon on/off one sun illumination.

To better understand the charge transport behavior within the $\mathrm{CN}$ electrode, we measured the photocurrent upon back and front illumination. These measurements 
reveal that the current upon front illumination is $\sim 70 \mu \mathrm{A} / \mathrm{cm}^{2}$ (Figure $4 \mathrm{~b}$ ), lower than the one observed when illuminating from the backside. The lower photocurrent may stem from light absorption from the flower-like structures upon front illumination and the slightly high electrode thickness. Nevertheless, it is still 140 times higher than that of the $\mathrm{CN}$ powder electrode, witnessing the advantageous charge separation and electron mobility within the $\mathrm{CN}$ film. The hole-extraction properties were assessed by measuring the photocurrent in the presence of triethanolamine (TEOA), an efficient hole scavenger. We assume that the photocurrent in presence of TEOA represents the maximum current that can be obtained without any hole-transfer limitations. ${ }^{25}$ The $\mathrm{CN}$ electrode exhibits a double photocurrent at $245 \mu \mathrm{A} / \mathrm{cm}^{2}$ after addition of $10 \%$ TEOA into the $0.1 \mathrm{M} \mathrm{KOH}$ solution (Figure S24a). In good agreement with this observation, the IPCE value at $400 \mathrm{~nm}$ is improved to $19 \%$ (Figure S24b). This means that the extraction efficiency of photogenerated holes in $0.1 \mathrm{M} \mathrm{KOH}$ is very high, reaching up to $47 \%$, which is a very impressive number considering the sluggish reaction kinetics for water oxidation in general.
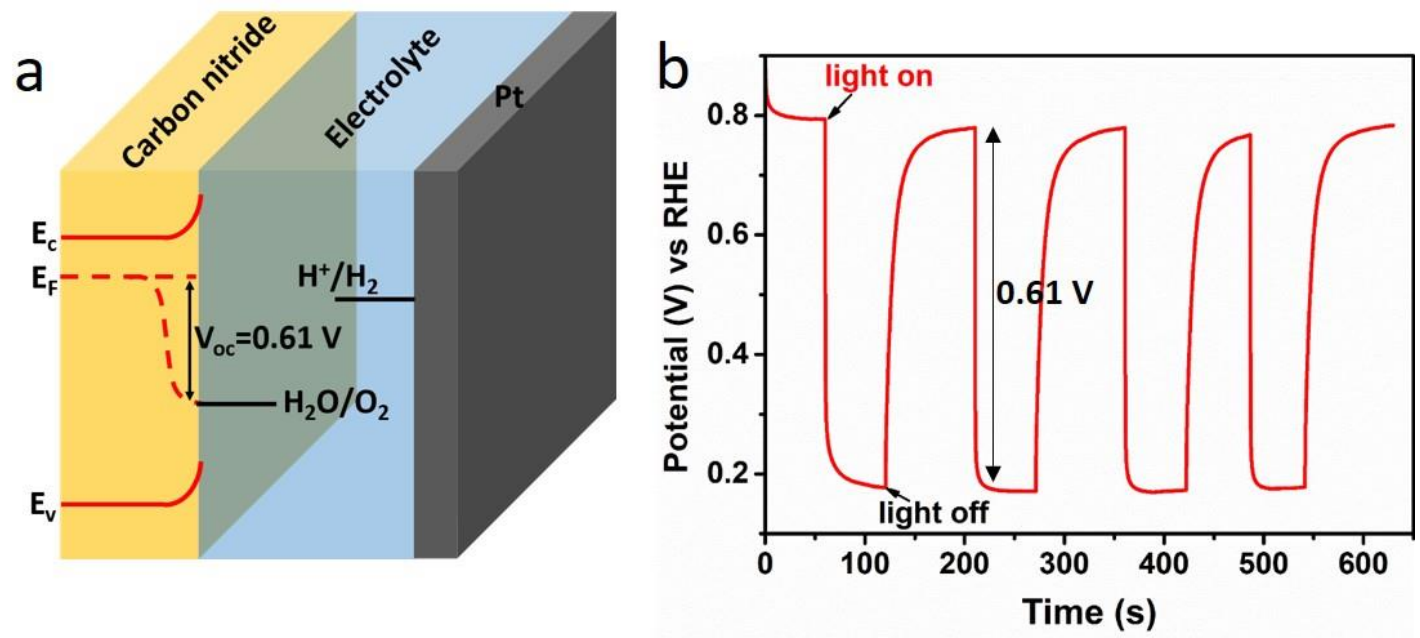

Figure 5. (a) A schematic illustration of the band diagram in the PEC cell. (b) Open circuit voltage of the $\mathrm{CN}$ electrode in $0.1 \mathrm{M} \mathrm{KOH}$ aqueous solution upon on/off one sun illumination. 
The good charge separation of photogenerated charges is supported by the high open-circuit voltages $\left(\mathrm{V}_{\mathrm{oc}}\right)$ of the $\mathrm{CN}$ electrode (Figure 5), which reflects the strong electron-hole separation driving force. The $\mathrm{V}_{\mathrm{oc}}$ in darkness corresponds to the Fermi level when in equilibrium with the electrolyte (Figure 5a). ${ }^{49,50} 51,52$ Under illumination, holes, the minority carriers in $\mathrm{CN}$, migrate to the $\mathrm{CN}$ surface and electrons, the majority carriers, are located near the $\mathrm{CN}$, leading to a $\mathrm{V}_{\mathrm{oc}}$ of $0.61 \mathrm{~V}$ (Figure $5 \mathrm{~b}$ ), while that of the $\mathrm{CN}$ powder electrode is negligible with a value lower than $0.02 \mathrm{~V}$ (Figure S25). The high $\mathrm{V}_{\mathrm{oc}}$ confirms the advantages of reduced grain boundaries in the continuous structure on the $\mathrm{CN}$ film. The negative shift of $\mathrm{V}_{\mathrm{oc}}$ upon illumination reflects the n-type semiconductor property of the $\mathrm{CN}$ film, while the fast $\mathrm{V}_{\mathrm{oc}}$ changes upon on/off illumination indicates the low amount of defect states in the $\mathrm{CN}$ film. Cyclic voltammetry $(\mathrm{CV})$ at negative potentials further confirmed the low level of structural defects, showing a very low capacitance, which is usually attributed to electrons in deep trap states within the $\mathrm{CN}$ film (Figure S26). Thus, electrons can freely move through the $\mathrm{CN}$ layer without being trapped and recombined. ${ }^{39}$ Electrochemical impedance spectroscopy (EIS) at $1.23 \mathrm{~V}$ vs. RHE reveals a charge transfer resistance that is seven times smaller than that of the $\mathrm{CN}$ powder electrode (Figure S27).

a)
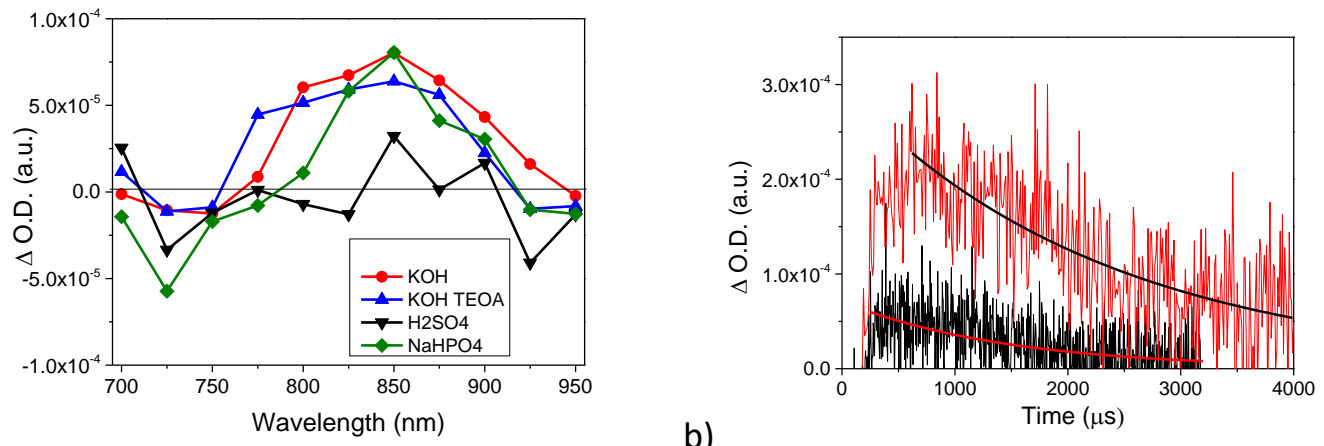

Figure 6a. (a) Transient absorption spectra of a CN film soaked in $0.1 \mathrm{M} \mathrm{KOH}, 0.1 \mathrm{M}$ KOH containing 10\% TEOA, $0.5 \mathrm{M} \mathrm{H}_{2} \mathrm{SO}_{4}$, and $0.1 \mathrm{M} \mathrm{NaH}_{2} \mathrm{PO}_{4}(\mathrm{pH}$ 7) aqueous solutions. (b) Transient absorption decay of a CN film soaked in $0.1 \mathrm{M} \mathrm{KOH}$ upon front 
(black) and back (red) excitation of a laser pulse and transient signals monitored at 850 nm. Laser excitation $355 \mathrm{~nm}$. Pt wire counter electrode.

Transient absorption spectroscopy measurements (TAS) of the $\mathrm{CN}$ film in various electrolytes at $1.23 \mathrm{~V}$ vs RHE were used to elucidate the charge separation mechanism, electron lifetime, and hole extraction kinetics (Figure 6). It is worthy noticing that TAS measurements without the applied $1.23 \mathrm{~V}$ vs RHE produced undetectable transient signal, indicating that charge recombination of photo-induced geminate elecvtron/hole pairs should take place faster than our instrument response $(1 \mu \mathrm{s})$. In contrast, applying $1.23 \mathrm{~V}$ vs RHE bias potential to $\mathrm{CN}$ films in contact with different electrolytes, a longlived transient signal could be recorded at $1 \mathrm{~ms}$ upon $355 \mathrm{~nm}$ laser flash excitation (Figure 6a). This TAS can be assigned to electrons in $\mathrm{CN}$ layer as reported before. ${ }^{39,53-}$ ${ }^{56}$ In good agreement with the photocurrent measurements, the electrons lifetime is longer at high $\mathrm{pH}$ due to a facilitated hole removal. Furthermore, the presence of TEOA as a hole scavenger further increases the electron lifetime, as a result of a faster hole extraction which disfavors electron-hole recombination (Table S3). Still, even in the absence of a hole scavenger, the electron lifetime in polarized $\mathrm{CN}$ films is extremely long thanks to an efficient hole extraction, decreasing its population in the $\mathrm{CN}$ layer and therefore diminishing the probability of electron-hole recombination (Figure S28 and Table S3). Moreover, larger transient intensity was observed for the back laser flash excitation compared to the front excitation of the $\mathrm{CN}$ films, as can be seen in Figure $6 \mathrm{~b}$. This larger electron population in the back irradiation is in good agreement with photocurrent measurements of Figure $4 \mathrm{~b}$ previously commented.

All the above-mentioned insights suggest that the good PEC performance is due to enhanced charge separation, electronic conductivity, and efficient hole extraction at the CN/electrolyte interface. The good hole-extraction properties can be attributed to the 
low recombination rate, fewer defect sites, as well as a layer porosity that is sufficient for good electrolyte penetration. Furthermore, because the electron and hole mobility within the $\mathrm{CN}$ layer is perpendicular to the $2 \mathrm{D}$ layer (unlike in graphene), ${ }^{38}$ the ordered sheet structure with closer layers should augment the electron and hole mobility.

\section{Conclusion}

We demonstrated a unique, simple and general method to grow highly ordered $\mathrm{CN}$ layer with a low amount of defects on conductive substrates from the seeded crystallization of a $\mathrm{CN}$ monomer. To initiate the crystallization process, a seeding layer of melamine on FTO is first deposited, followed by the immersion of the seeded substrate into a supersaturated solution of melamine at high temperature. Upon cooling, melamine crystals spontaneously grew on the seeded substrate to form a well-covered melamine film. Upon calcination of the melamine film at high temperature, a strongly attached and crystalline $\mathrm{CN}$ layer with an interconnected, porous structure is synthesized with an intimate contact with the substrate. The closer stacking of the layers permits better electron and hole mobility, which significantly decreases the recombination rate, thereby elongating the lifetime of photogenerated electrons and holes, and increasing their probability to reach the conductive substrate and react with the electrolyte, respectively. Detailed photoelectrochemical and photophysical measurements reveal an excellent hole-extraction efficiency $(\sim 50 \%)$, and confirm the long electron lifetime, good charges conductivity and fast charge separation under illumination. Consequently, the $\mathrm{CN}$ photoanode exhibits an exceptionally low onset potential (up to $1 \mathrm{~V}$ shift) which is comparable to the best metal-based photoanodes, a good photocurrent density, and an external quantum efficiency of $\sim 8.5 \%$ at $400 \mathrm{~nm}$, as well as good stability over a wide $\mathrm{pH}$ range (0-13). 


\section{Acknowledgements.}

JA thanks the Technical University of Valencia for a postdoctoral research associate contract.

\section{References}

1. Wang, X.; Maeda, K.; Thomas, A.; Takanabe, K.; Xin, G.; Carlsson, J. M.; Domen, K.; Antonietti, M., A metal-free polymeric photocatalyst for hydrogen production from water under visible light. Nature materials 2009, 8, 76-80.

2. Lau, V. W.; Moudrakovski, I.; Botari, T.; Weinberger, S.; Mesch, M. B.; Duppel, V.; Senker, J.; Blum, V.; Lotsch, B. V., Rational design of carbon nitride photocatalysts by identification of cyanamide defects as catalytically relevant sites. Nat Commun 2016, 7, 12165. 3. Yang, L.; Li, X.; Zhang, G.; Cui, P.; Wang, X.; Jiang, X.; Zhao, J.; Luo, Y.; Jiang, J., Combining photocatalytic hydrogen generation and capsule storage in graphene based sandwich structures. Nat Commun 2017, 8, 16049.

4. Ran, J.; Ma, T. Y.; Gao, G.; Du, X.-W.; Qiao, S. Z., Porous P-doped graphitic carbon nitride nanosheets for synergistically enhanced visible-light photocatalytic $\mathrm{H} 2$ production. Energy \& Environmental Science 2015, 8, 3708-3717.

5. Zhu, M.; Kim, S.; Mao, L.; Fujitsuka, M.; Zhang, J.; Wang, X.; Majima, T., Metal-Free Photocatalyst for H2 Evolution in Visible to Near-Infrared Region: Black Phosphorus/Graphitic Carbon Nitride. Journal of the American Chemical Society 2017, 139, 13234-13242.

6. Ran, J.; Guo, W.; Wang, H.; Zhu, B.; Yu, J.; Qiao, S. Z., Metal-Free 2D/2D Phosphorene/g-C3 N4 Van der Waals Heterojunction for Highly Enhanced Visible-Light Photocatalytic H2 Production. Advanced materials 2018, 30, e1800128.

7. Schwinghammer, K.; Mesch, M. B.; Duppel, V.; Ziegler, C.; Senker, J.; Lotsch, B. V., Crystalline carbon nitride nanosheets for improved visible-light hydrogen evolution. Journal of the American Chemical Society 2014, 136, 1730-3.

8. She, X.; Wu, J.; Xu, H.; Zhong, J.; Wang, Y.; Song, Y.; Nie, K.; Liu, Y.; Yang, Y.; Rodrigues, M.-T. F.; Vajtai, R.; Lou, J.; Du, D.; Li, H.; Ajayan, P. M., High Efficiency Photocatalytic Water Splitting Using 2D a-Fe2O3/g-C3N4 Z-Scheme Catalysts. Advanced Energy Materials 2017, 7, 1700025.

9. $\quad$ Zheng, Y.; Jiao, Y.; Zhu, Y.; Cai, Q.; Vasileff, A.; Li, L. H.; Han, Y.; Chen, Y.; Qiao, S. Z., Molecule-Level g-C3N4 Coordinated Transition Metals as a New Class of Electrocatalysts for Oxygen Electrode Reactions. Journal of the American Chemical Society 2017, 139, 3336-3339.

10. Yang, S.; Feng, X.; Wang, X.; Mullen, K., Graphene-based carbon nitride nanosheets as efficient metal-free electrocatalysts for oxygen reduction reactions. Angewandte Chemie 2011, 50, 5339-43.

11. Ma, T. Y.; Ran, J.; Dai, S.; Jaroniec, M.; Qiao, S. Z., Phosphorus-doped graphitic carbon nitrides grown in situ on carbon-fiber paper: flexible and reversible oxygen electrodes. Angewandte Chemie 2015, 54, 4646-50.

12. <Carbon Nitride as a Nonprecious Catalyst for Electrochemical Oxygen Reduction jp907928j.pdf>.

13. Cao, S.; Li, Y.; Zhu, B.; Jaroniec, M.; Yu, J., Facet effect of Pd cocatalyst on photocatalytic CO 2 reduction over g-C 3 N 4. Journal of Catalysis 2017, 349, 208-217.

14. Kuriki, R.; Sekizawa, K.; Ishitani, O.; Maeda, K., Visible-light-driven $\mathrm{CO} 2$ reduction with carbon nitride: enhancing the activity of ruthenium catalysts. Angewandte Chemie 2015, 54, 2406-9. 
15. Gao, G.; Jiao, Y.; Waclawik, E. R.; Du, A., Single Atom (Pd/Pt) Supported on Graphitic Carbon Nitride as an Efficient Photocatalyst for Visible-Light Reduction of Carbon Dioxide. Journal of the American Chemical Society 2016, 138, 6292-7.

16. Jiang, Z.; Wan, W.; Li, H.; Yuan, S.; Zhao, H.; Wong, P. K., A Hierarchical Z-Scheme alpha-Fe2 $\mathrm{O} 3$ /g-C3 N4 Hybrid for Enhanced Photocatalytic CO2 Reduction. Advanced materials 2018, 30.

17. Xia, P.; Zhu, B.; Yu, J.; Cao, S.; Jaroniec, M., Ultra-thin nanosheet assemblies of graphitic carbon nitride for enhanced photocatalytic $\mathrm{CO} 2$ reduction. Journal of Materials Chemistry A 2017, 5, 3230-3238.

18. Fu, J.; Zhu, B.; Jiang, C.; Cheng, B.; You, W.; Yu, J., Hierarchical Porous O-Doped g-C3 N4 with Enhanced Photocatalytic CO2 Reduction Activity. Small 2017, 13.

19. Wang, Y.; Wang, X.; Antonietti, M., Polymeric graphitic carbon nitride as a heterogeneous organocatalyst: from photochemistry to multipurpose catalysis to sustainable chemistry. Angewandte Chemie 2012, 51, 68-89.

20. <ANanojunctionPolymerPhotoelectrodefor EfficientChargeTransportand Separation Ruan_et_al-2017-Angewandte_Chemie_International_Edition.pdf>.

21. Bian, J.; Li, Q.; Huang, C.; Li, J.; Guo, Y.; Zaw, M.; Zhang, R.-Q., Thermal vapor condensation of uniform graphitic carbon nitride films with remarkable photocurrent density for photoelectrochemical applications. Nano Energy 2015, 15, 353-361.

22. Bian, J.; Xi, L.; Huang, C.; Lange, K. M.; Zhang, R.-Q.; Shalom, M., Efficiency Enhancement of Carbon Nitride Photoelectrochemical Cells via Tailored Monomers Design. Advanced Energy Materials 2016, 6, 1600263.

23. Liu, J.; Wang, H.; Chen, Z. P.; Moehwald, H.; Fiechter, S.; van de Krol, R.; Wen, L.; Jiang, L.; Antonietti, M., Microcontact-printing-assisted access of graphitic carbon nitride films with favorable textures toward photoelectrochemical application. Advanced materials 2015, 27, 712-8.

24. Peng, G.; Xing, L.; Barrio, J.; Volokh, M.; Shalom, M., A General Synthesis of Porous Carbon Nitride Films with Tunable Surface Area and Photophysical Properties. Angewandte Chemie 2018, 57, 1186-1192.

25. Peng, G.; Volokh, M.; Tzadikov, J.; Sun, J.; Shalom, M., Carbon Nitride/Reduced Graphene Oxide Film with Enhanced Electron Diffusion Length: An Efficient PhotoElectrochemical Cell for Hydrogen Generation. Advanced Energy Materials 2018, 1800566.

26. <Activation of Carbon Nitride Solids by Protonation Morphology Changes Enhanced Ionic Conductivity and Photoconduction Experiments ja808329f_si_001.pdf $>$.

27. Lou, S.; Zhou, Z.; Shen, Y.; Zhan, Z.; Wang, J.; Liu, S.; Zhang, Y., Comparison Study of the Photoelectrochemical Activity of Carbon Nitride with Different Photoelectrode Configurations. ACS applied materials \& interfaces 2016, 8, 22287-94.

28. Zhang, J.; Zhang, M.; Lin, L.; Wang, X., Sol processing of conjugated carbon nitride powders for thin-film fabrication. Angewandte Chemie 2015, 54, 6297-301.

29. Xu, J.; Brenner, T. J.; Chabanne, L.; Neher, D.; Antonietti, M.; Shalom, M., Liquid-based growth of polymeric carbon nitride layers and their use in a mesostructured polymer solar cell with V(oc) exceeding 1 V. Journal of the American Chemical Society 2014, 136, 13486-9.

30. Shalom, M.; Gimenez, S.; Schipper, F.; Herraiz-Cardona, I.; Bisquert, J.; Antonietti, M., Controlled carbon nitride growth on surfaces for hydrogen evolution electrodes. Angewandte Chemie 2014, 53, 3654-8.

31. <Carbon Nitride Materials for Water Splitting Photoelectrochemical Cells Volokh_et_al-2018-Angewandte_Chemie_International_Edition.pdf $>$.

32. Zhang, X.; Zheng, Q.; Tang, Z.; Li, W.; Zhang, Y.; Xu, K.; Xue, X.; Xu, J.; Wang, H.; Wei, B., Tunable hole injection of solution-processed polymeric carbon nitride towards efficient organic light-emitting diode. Applied Physics Letters 2018, 112, 083302. 
33. Xu, J.; Shalom, M.; Piersimoni, F.; Antonietti, M.; Neher, D.; Brenner, T. J. K., ColorTunable Photoluminescence and NIR Electroluminescence in Carbon Nitride Thin Films and Light-Emitting Diodes. Advanced Optical Materials 2015, 3, 913-917.

34. Gan, Z.; Shan, Y.; Chen, J.; Gui, Q.; Zhang, Q.; Nie, S.; Wu, X., The origins of the broadband photoluminescence from carbon nitrides and applications to white light emitting. Nano Research 2016, 9, 1801-1812.

35. Jiang, L.-L.; Wang, Z.-K.; Li, M.; Zhang, C.-C.; Ye, Q.-Q.; Hu, K.-H.; Lu, D.-Z.; Fang, P.-F.; Liao, L.-S., Passivated Perovskite Crystallization via g-C3N4 for High-Performance Solar Cells. Advanced Functional Materials 2018, 28, 1705875.

36. Chen, X.; Liu, Q.; Wu, Q.; Du, P.; Zhu, J.; Dai, S.; Yang, S., Incorporating Graphitic Carbon Nitride (g-C3N4) Quantum Dots into Bulk-Heterojunction Polymer Solar Cells Leads to Efficiency Enhancement. Advanced Functional Materials 2016, 26, 1719-1728.

37. Li, X.; Pan, K.; Qu, Y.; Wang, G., One-dimension carbon self-doping g-C3N4 nanotubes: Synthesis and application in dye-sensitized solar cells. Nano Research 2018, 11, 1322-1330.

38. Merschjann, C.; Tschierlei, S.; Tyborski, T.; Kailasam, K.; Orthmann, S.; Hollmann, D.; Schedel-Niedrig, T.; Thomas, A.; Lochbrunner, S., Complementing Graphenes: 1D Interplanar Charge Transport in Polymeric Graphitic Carbon Nitrides. Advanced materials 2015, 27, 79939.

39. Godin, R.; Wang, Y.; Zwijnenburg, M. A.; Tang, J.; Durrant, J. R., Time-Resolved Spectroscopic Investigation of Charge Trapping in Carbon Nitrides Photocatalysts for Hydrogen Generation. Journal of the American Chemical Society 2017, 139, 5216-5224.

40. Zhang, J.; Zhang, M.; Zhang, G.; Wang, X., Synthesis of Carbon Nitride Semiconductors in Sulfur Flux for Water Photoredox Catalysis. ACS Catalysis 2012, 2, 940-948.

41. Shalom, M.; Inal, S.; Fettkenhauer, C.; Neher, D.; Antonietti, M., Improving carbon nitride photocatalysis by supramolecular preorganization of monomers. Journal of the American Chemical Society 2013, 135, 7118-21.

42. Chen, Y.; Wang, B.; Lin, S.; Zhang, Y.; Wang, X., Activation of $n \rightarrow \pi *$ Transitions in Two-Dimensional Conjugated Polymers for Visible Light Photocatalysis. The Journal of Physical Chemistry C 2014, 118, 29981-29989.

43. Thomas, A.; Fischer, A.; Goettmann, F.; Antonietti, M.; Müller, J.-O.; Schlögl, R.; Carlsson, J. M., Graphitic carbon nitride materials: variation of structure and morphology and their use as metal-free catalysts. Journal of Materials Chemistry 2008, 18, 4893.

44. Xu, J.; Wang, H.; Zhang, C.; Yang, X.; Cao, S.; Yu, J.; Shalom, M., From Millimeter to Subnanometer: Vapor-Solid Deposition of Carbon Nitride Hierarchical Nanostructures Directed by Supramolecular Assembly. Angewandte Chemie 2017, 56, 8426-8430.

45. Xiong, W.; Chen, S.; Huang, M.; Wang, Z.; Lu, Z.; Zhang, R. Q., Crystal-Face Tailored Graphitic Carbon Nitride Films for High-Performance Photoelectrochemical Cells. ChemSusChem 2018.

46. Lee, D. K.; Choi, K.-S., Enhancing long-term photostability of BiVO4 photoanodes for solar water splitting by tuning electrolyte composition. Nature Energy 2017, 3, 53-60.

47. Kuang, Y.; Jia, Q.; Ma, G.; Hisatomi, T.; Minegishi, T.; Nishiyama, H.; Nakabayashi, M.; Shibata, N.; Yamada, T.; Kudo, A.; Domen, K., Ultrastable low-bias water splitting photoanodes via photocorrosion inhibition and in situ catalyst regeneration. Nature Energy 2016, 2, 16191. 48. Kim, T. W.; Ping, Y.; Galli, G. A.; Choi, K. S., Simultaneous enhancements in photon absorption and charge transport of bismuth vanadate photoanodes for solar water splitting. Nat Commun 2015, 6, 8769.

49. Hajduk, Š.; Berglund, S. P.; Podlogar, M.; Dražić, G.; Abdi, F. F.; Orel, Z. C.; Shalom, M., Conformal Carbon Nitride Coating as an Efficient Hole Extraction Layer for ZnO NanowiresBased Photoelectrochemical Cells. Advanced Materials Interfaces 2017, 4, 1700924. 
50. Jiang, C.; Moniz, S. J. A.; Wang, A.; Zhang, T.; Tang, J., Photoelectrochemical devices for solar water splitting - materials and challenges. Chemical Society reviews 2017, 46, 46454660.

51. Hara, K. O.; Usami, N., Theory of open-circuit voltage and the driving force of charge separation in pn-junction solar cells. Journal of Applied Physics 2013, 114, 153101.

52. <Photovoltaic Technology The Case for Thin-Film Solar Cells 692.full.pdf>.

53. Albero, J.; Barea, E. M.; Xu, J.; Mora-Seró, I.; Garcia, H.; Shalom, M., Toward Efficient Carbon Nitride Photoelectrochemical Cells: Understanding Charge Transfer Processes. Advanced Materials Interfaces 2017, 4, 1600265.

54. Kasap, H.; Caputo, C. A.; Martindale, B. C.; Godin, R.; Lau, V. W.; Lotsch, B. V.; Durrant, J. R.; Reisner, E., Solar-Driven Reduction of Aqueous Protons Coupled to Selective Alcohol Oxidation with a Carbon Nitride-Molecular Ni Catalyst System. Journal of the American Chemical Society 2016, 138, 9183-92.

55. Kuriki, R.; Matsunaga, H.; Nakashima, T.; Wada, K.; Yamakata, A.; Ishitani, O.; Maeda, K., Nature-Inspired, Highly Durable CO2 Reduction System Consisting of a Binuclear Ruthenium(II) Complex and an Organic Semiconductor Using Visible Light. Journal of the American Chemical Society 2016, 138, 5159-70.

56. Zhang, W.; Albero, J.; Xi, L.; Lange, K. M.; Garcia, H.; Wang, X.; Shalom, M., One-Pot Synthesis of Nickel-Modified Carbon Nitride Layers Toward Efficient Photoelectrochemical Cells. ACS applied materials \& interfaces 2017, 9, 32667-32677. 